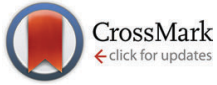

Cite this: J. Mater. Chem. C, 2015 3, 10459

\title{
Mechanochemical synthesis and high temperature thermoelectric properties of calcium-doped lanthanum telluride $\mathrm{La}_{3-x} \mathrm{Ca}_{x} \mathrm{Te}_{4}$
}

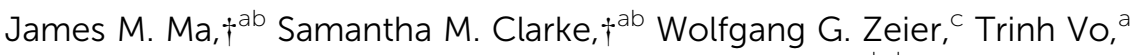 \\ Paul Von Allmen, ${ }^{a}$ G. Jeffrey Snyder, ${ }^{c}$ Richard B. Kaner, ${ }^{\text {bd }}$ Jean-Pierre Fleurial ${ }^{a}$ and \\ Sabah K. Bux $\star^{\star^{a}}$
}

\begin{abstract}
The thermoelectric properties from $300-1275 \mathrm{~K}$ of calcium-doped $\mathrm{La}_{3-x} \mathrm{Te}_{4}$ are reported. $\mathrm{La}_{3-x} \mathrm{Te}_{4}$ is a high temperature $\mathrm{n}$-type thermoelectric material with a previously reported $\mathrm{zT}_{\max } \sim 1.1$ at $1273 \mathrm{~K}$ and $x=0.23$. Computational modeling suggests the La atoms define the density of states of the conduction band for $\mathrm{La}_{3-x} \mathrm{Te}_{4}$. Doping with $\mathrm{Ca}^{2+}$ on the $\mathrm{La}^{3+}$ site is explored as a means of modifying the density of states to improve the power factor and to achieve a finer control over the carrier concentration. High purity, oxide-free samples are produced by ball milling of the elements and consolidation by spark plasma sintering. Calcium substitution upon the lanthanum site was confirmed by a combination of Rietveld refinements of powder $\mathrm{X}$-ray diffraction data and wave dispersive spectroscopy. $A \mathrm{zT}_{\max } \sim 1.2$ is reached at $1273 \mathrm{~K}$ for the composition $\mathrm{La}_{2.2} \mathrm{Ca}_{0.78} \mathrm{Te}_{4}$ and the relative increase compared to $\mathrm{La}_{3-x} \mathrm{Te}_{4}$ is attributed to the finer carrier concentration.
\end{abstract}

Received 4th June 2015

Accepted 22nd June 2015

DOI: $10.1039 / \mathrm{c} 5 \mathrm{tc} 01648 \mathrm{~g}$

www.rsc.org/MaterialsC

\section{Introduction}

Thermoelectric (TE) materials are solid state energy conversion devices which are able to generate electricity through the Seebeck effect or serve as a heat pump through the Peltier effect. Thermoelectric generators (TEGs) have demonstrated their extraordinary reliability and longevity in support of the National Aeronautics Space Administration's (NASA) deep space science and exploration missions. The TEGs powering the Voyager missions launched in 1977 are still operational. ${ }^{1}$ Most recently, a TEG powers the Curiosity rover on Mars with TEGs continuing to be a key technology for future missions. ${ }^{2}$

State-of-practice TEGs have system level thermal-to-electric energy conversion efficiencies of 6 to $6.5 \%$ utilizing materials with a dimensionless thermoelectric figure of merit zT close to $1 .^{3,4}$ The zT is defined as zT $=S^{2} T /(\rho \kappa)$ and good TE materials exhibit low thermal conductivity $(\kappa)$, low electrical resistivity $(\rho)$,

\footnotetext{
${ }^{a}$ Thermal Energy Conversion Technologies, Jet Propulsion Laboratory, California Institute of Technology, 4800 Oak Grove Drive, Pasadena, California 91109-8099, USA. E-mail: sabah.k.bux@jpl.nasa.gov

${ }^{b}$ Department of Materials Science and Engineering, University of California, Los Angeles, 90092, USA

${ }^{c}$ Department of Material Science and Engineering, Northwestern University, Evanston, IL 60208, USA

${ }^{d}$ Department of Chemistry and Biochemistry, University of California, Los Angeles, 90092, USA

$\dagger$ These authors contributed equally to this work.
}

and a large Seebeck coefficient $(S)$. The thermal conductivity is the sum of the electronic contribution, $\kappa_{\mathrm{e}}$, and the lattice contribution, $\kappa_{\mathrm{L}}$. Further, the ultimate efficiency of the TE device is dependent upon the zT across the temperature range of operation, $T_{\max }$, and $\Delta T$ thereby making high temperature materials desirable to maximize efficiency. New materials with improved zT are sought to reduce the weight of the spacecraft, enable additional instrumentation, and reduce $\mathrm{Pu}-238$ fuel requirements. ${ }^{5}$

Recent development of new thermoelectric materials has doubled the TE couple-level efficiency to $15 \%$ roughly doubling heritage technologies. ${ }^{6}$ The high temperature (875-1273 K) n-type segment of this high efficiency prototype is lanthanum telluride, $\mathrm{La}_{3-x} \mathrm{Te}_{4}$, a self-doped refractory rare earth chalcogenide with a reported zT of 1.1 at $1273 \mathrm{~K}$ when $x=0.23 .^{7,8}$

The compound, $\mathrm{La}_{3-x} \mathrm{Te}_{4}$, has a defect structure with the carrier concentration varied through controlling the lanthanum vacancy concentration. It can be understood as each lanthanum atom contributing 3 electrons and 2 electrons are required for each tellurium atom. Thereby, the electronic properties can vary between metallic at $x=0$ with one free $\mathrm{e}^{-}$per formula unit $\left(\mathrm{La}_{3} \mathrm{Te}_{4}\right)$ to an insulator at $x=0.33$ with no free carriers $\left(\mathrm{La}_{2.67} \mathrm{Te}_{4}\right)$. In addition, the material has good electronic transport properties due to the favorable band structure of the material. ${ }^{9}$ The existence of heavy conduction bands allows for high Seebeck coefficients even at high carrier concentrations. Lastly, $\mathrm{La}_{3-x} \mathrm{Te}_{4}$ exhibits low lattice thermal conductivity due to the complex crystal structure, phonon scattering from the vacancies, and electron-phonon 
scattering at high carrier concentrations. ${ }^{9,10}$ This results in the high $\mathrm{zT}=1.1$ at $1273 \mathrm{~K}$ at the optimum carrier concentration $n \sim 0.9 \times 10^{20} \mathrm{~cm}^{-3}$.

From computational modelling, it has been found that the density of the states (DOS) in the conduction band is dominated by the $5 \mathrm{~d}$ orbitals from the lanthanum atoms., ${ }^{9,11}$ Non-isoelectronic cation substitutions $\mathrm{M}^{2+}$ on the $\mathrm{La}^{3+}$ site could increase the Seebeck coefficient for this material through a modification of the density of states. In addition, the use of a divalent cation would allow for a finer control over the carrier concentration. The electronic local environment becomes $\mathrm{La}_{3-x-y}^{3+} \square_{\mathrm{La}, x} \mathrm{M}_{y}^{2+} \mathrm{Te}_{4}^{2-} \mathrm{e}_{1-3 x}^{1-}$, where $\square_{\mathrm{La}, x}$ represents the number of vacancies in the system and $\mathbf{M}_{y}^{2+}$ represents the amount of non-isoelectronic metal substituents in the system. The theoretical carrier density can be calculated by $n=n_{\max }(1-3 x-y)$ where $n_{\max }=4.5 \times 10^{21} \mathrm{~cm}^{-3}$ and is the carrier concentration of the metallic system, $x$ is the number of lanthanum vacancies, and $y$ is the number of divalent cations. ${ }^{12}$ There is a threefold finer control of carrier concentration in the $\mathrm{M}^{2+}$ doped system compared to the vacancy-doped system. Hence, a divalent cation substitution allows for more precise synthesis of samples with carrier concentrations in the optimized region of $0.8 \times 10^{21} \mathrm{~cm}^{-3}$ to $1.2 \times 10^{21} \mathrm{~cm}^{-3}$, which had previously been difficult to obtain. ${ }^{13}$ This doping also provides a way to create vacancy-free structures with identical carrier concentrations to the vacancy-doped samples, allowing for a separation of the impact on the lattice thermal conductivity of various defects on the La sublattice (vacancies, divalent atoms) versus electron-phonon interactions.

For this study, calcium was chosen as the dopant because of the definitive $2+$ charge, similarity in size to $\mathrm{La}^{3+}$ which enhances the probability of successful substitution upon that site, and the difference in electronic properties from lanthanum atoms as it lacks electrons in the $\mathrm{d}$ orbital. Non-isoelectronic cation substitution has been previously explored with ytterbium, but the cation can be mixed valence $\mathrm{Yb}^{2+} / \mathrm{Yb}^{3+}$ and is also a rare earth similar to lanthanum. ${ }^{12}$ Substitution with a non-rare earth dopant is pursued here as it can result in larger changes to the DOS by introducing a different chemical bonding environment. In this study, the calcium-doped compounds are vacancy-free to prevent conflation of the effects of calcium versus vacancies.

\section{Results and discussion}

\section{Sample characterization}

The samples were analyzed for phase purity through powder X-ray diffraction. An exemplary diffraction pattern of $\mathrm{La}_{3-x} \mathrm{Ca}_{x} \mathrm{Te}_{4}$ is shown in Fig. 1. The diffraction pattern indicates there is good agreement to the phase for $\mathrm{La}_{3-x} \mathrm{Te}_{4}$ as established by Cox et al. ${ }^{14}$ The residual intensity is low and the samples are phase pure with no secondary or oxide phases present. The refinement parameters are presented in Table 1 . The low values for the goodness-of-fit indicate good agreement with the proposed model. Changes in the Te position account for the observed lattice expansion only.

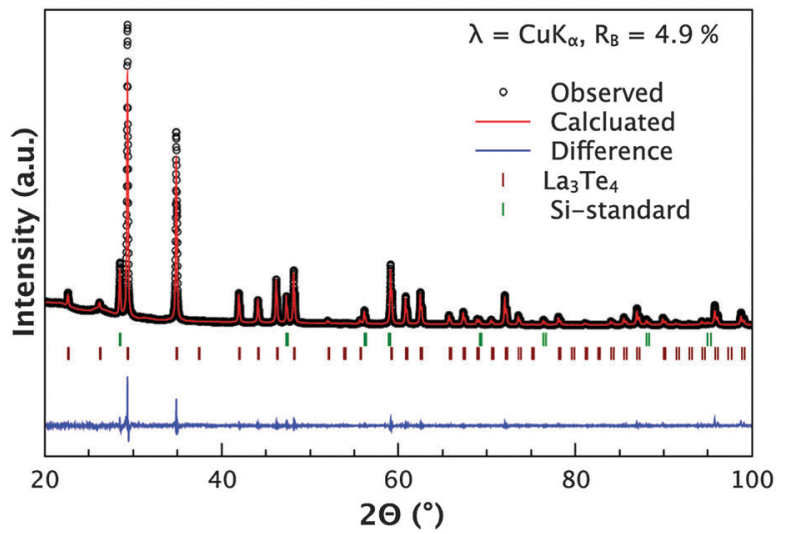

Fig. 1 X-ray diffraction pattern of $\mathrm{La}_{2.2} \mathrm{Ca}_{0.8} \mathrm{Te}_{4}$ including profile fit, profile difference, and profile residuals of the corresponding Rietveld refinement, showing a good match to the $\mathrm{La}_{3} \mathrm{Te}_{4}$ phase. ${ }^{14}$ The diffraction pattern is representative of other compositions. A silicon internal standard was used and the reflections are indicated. There are no oxide or secondary phases present.

Table 1 Relevant crystallographic information determined from Rietveld refinement of the solid solutions $\mathrm{La}_{3-x} \mathrm{Ca}_{x} \mathrm{Te}_{4} . R_{\mathrm{B}}$ is the Bragg residual, GoF is the goodness of fit. Listed are the refined unit cell parameter $a$, atomic positions $(x, y, z)$, isotropic atomic displacement parameters $\left(U_{\text {iso }}\right)$, and atomic occupancies

\begin{tabular}{lllll}
\hline $\mathrm{La}_{3-x} \mathrm{Ca}_{x} \mathrm{Te}_{4} I \overline{4} 3 d$ & $x=0$ & $x=0.609$ & $x=0.775$ & $x=0.8$ \\
\hline$R_{\mathrm{B}}(\%)$ & 5.1 & 4.8 & 7.9 & 4.9 \\
$\mathrm{GoF}$ & 5.3 & 3.6 & 2.2 & 4.2 \\
$a(\AA)$ & $9.626(1)$ & $9.628(1)$ & $9.632(1)$ & $9.630(1)$ \\
$\mathrm{La} / \mathrm{Ca}(x, y, z)$ & $(0.375,0,0.5)$ & $(0.375,0,0.5)$ & $(0.375,0,0.5)$ & $(0.375,0,0.5)$ \\
$\mathrm{Te}(x=y=z)$ & $0.07538(8)$ & $0.07386(6)$ & $0.07291(7)$ & $0.07320(7)$ \\
$U_{\text {iso }}(\mathrm{La} / \mathrm{Ca})\left(\AA^{2}\right)$ & $0.0282(4)$ & $0.0318(3)$ & $0.0335(5)$ & $0.0259(4)$ \\
$U_{\text {iso }}(\mathrm{Te})\left(\AA^{2}\right)$ & $0.0278(4)$ & $0.0335(3)$ & $0.0356(4)$ & $0.0259(4)$ \\
Occ(La) & $0.993(4)$ & $0.791(4)$ & $0.709(7)$ & $0.729(5)$ \\
Occ(Ca) & 0 & $0.209(4)$ & $0.291(7)$ & $0.271(5)$ \\
& & & &
\end{tabular}

It has been previously suggested through computational modeling that cation vacancy ordering can stabilize the structure by increasing the distance in between charge centers. ${ }^{15}$ Experimental results from the powder XRD suggest a random distribution of the $\mathrm{Ca}^{2+}$ atoms on the $\mathrm{La}^{3+}$ site for the polycrystalline sample, since no superstructure reflections were found.

Rietveld analysis is used to determine the composition of the samples through refinement of the calcium occupancy on the lanthanum position. There is good agreement between the nominal compositions and refined composition as seen in Fig. 2. The solubility limit of calcium in the $\mathrm{La}_{3-x} \mathrm{Te}_{4}$ was not fully explored due to the limited thermoelectric usefulness of very low carrier concentration samples and the difficulty of handling samples in air. It was observed that oxygen and moisture sensitivity of the samples appeared to increase with increasing calcium content although both lanthanum and calcium are oxygen and moisture reactive metals.

The lattice parameters of the calcium-doped samples were determined through XRD and the lattice parameter as a function of the refined composition is presented in Fig. 3. There is a small and steady increase in the lattice parameter as a function 


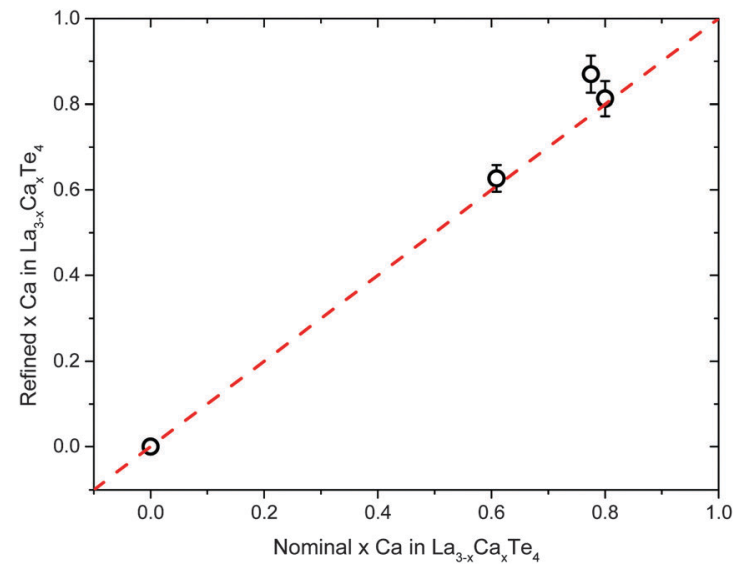

Fig. 2 The Rietveld refined composition of $\mathrm{La}_{3-x} \mathrm{Ca}_{x} \mathrm{Te}_{4}$ matches well to nominal compositions. Error bars represent a $5 \%$ uncertainty.

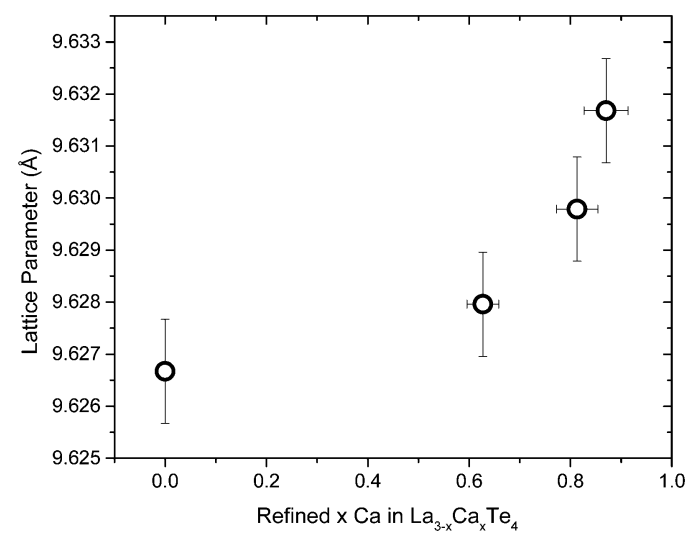

Fig. 3 The lattice parameter of the $\mathrm{La}_{3-x} \mathrm{Ca}_{x} \mathrm{Te}_{4}$ as a function of the refined calcium content. $Y$-error bars represent the instrumental limit of $\pm 0.001 \AA$ and $X$-error bars represent a $5 \%$ uncertainty.

of calcium doping. Even with a $\sim 30 \%$ substitution of calcium on the lanthanum position, the lattice parameter increases only by $0.003 \AA$. This is expected since the ionic radii of $\mathrm{Ca}^{2+}(114.0 \mathrm{pm})$ is similar in size to the ionic radii of $\mathrm{La}^{3+}(117.2 \mathrm{pm}) .{ }^{16}$ Therefore, the $\mathrm{Ca}^{2+}$ atom should be able to occupy a $\mathrm{La}^{3+}$ without much distortion to the lattice.

This result suggests that a variety of other cation dopants can occupy the $\mathrm{La}^{3+}$ position indicating a phase space rich with possibilities for cationic doping of $\mathrm{La}^{3+}$. The vacancy limit for $\mathrm{La}_{3-x} \mathrm{Te}_{4}$ is $x=0.33$ and it is possible to dope beyond this threshold with calcium atoms. Therefore the calcium atoms are more than just occupying vacancy positions within the $\mathrm{La}_{3-x} \mathrm{Te}_{4}$ network. The calcium atoms fully substitute the lanthanum position and help form the structure. This result is promising for modification of the electronic structure of $\mathrm{La}_{3-x} \mathrm{Te}_{4}$. It is interesting to note that calcium and tellurium have not been shown to create the $\mathrm{Th}_{3} \mathrm{P}_{4}$ structure type. Therefore this suggests that the $\mathrm{La}^{3+}$ in $\mathrm{La}_{3-x} \mathrm{Te}_{4}$ is amendable to doping by a wide variety of dopants which are chemically different in so far that charge balance is preserved or there is an excess of electrons. This is promising as it provides guidelines for future

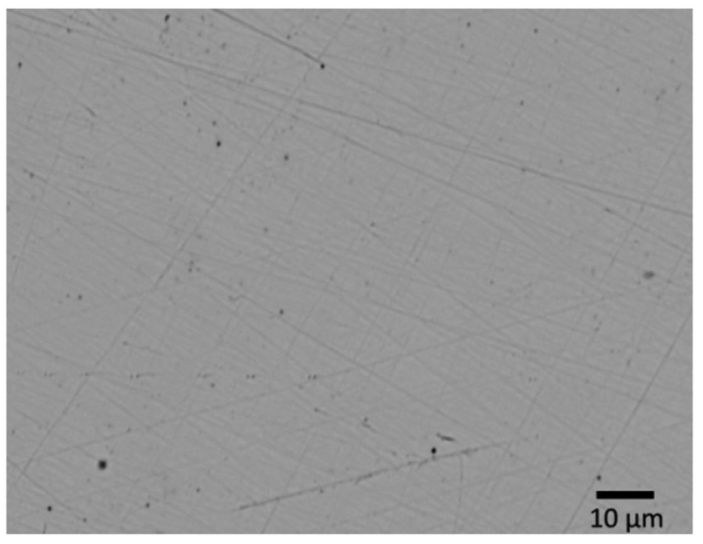

Fig. 4 Backscattered electron image of the polished surface of $\mathrm{La}_{2.22} \mathrm{Ca}_{0.78} \mathrm{Te}_{4}$. The image is representative of other compositions. The sample composition is uniform as reflected by the uniformity in image contrast. Fine scratches are observable and are difficult to fully remove by polishing due to the brittleness of the material.

substitutions by a wide variety of dopants which can modify the behavior of this already high zT material.

The calcium-substituted samples were found to be homogeneous as seen through the uniform contrast in the backscattered electron (BSE) micrograph of a typical sample (Fig. 4). The dark spots in the image are due to small amounts of porosity.

\section{Electronic transport properties}

In this study, compositions were studied near the previously optimized carrier concentration of $0.9 \times 10^{21} \mathrm{~cm}^{-3}$ for $\mathrm{La}_{3-x} \mathrm{Te}_{4}{ }^{3}$ The room temperature carrier concentrations of the samples measured through the Hall effect are compared to the carrier concentration calculated from the compositions determined by XRD and WDS (Fig. 5). There is good agreement between carrier concentrations as determined by the various methods confirming that the calcium is reliably doped into the system.

The low carrier mobility of $\mathrm{La}_{3-x} \mathrm{Ca}_{x} \mathrm{Te}_{4}<4 \mathrm{~cm}^{2} \mathrm{~V}^{-1} \mathrm{~s}^{-1}$ presents a challenge in the accurate measurement of the Hall

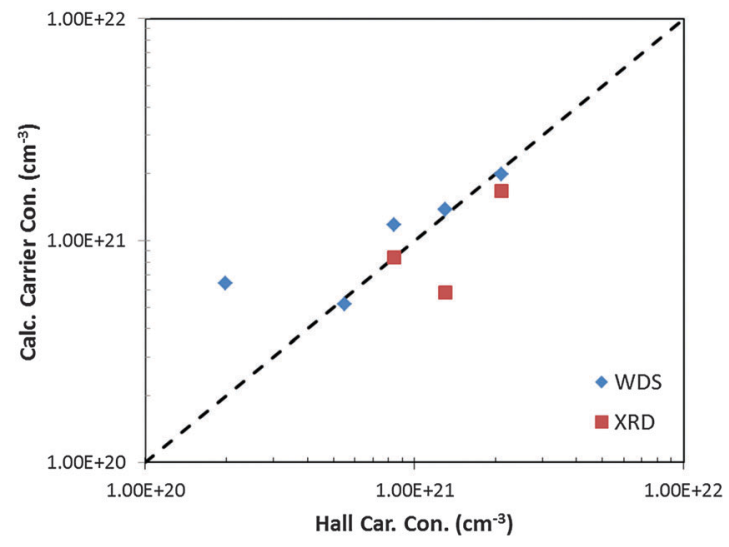

Fig. 5 The carrier concentration of $\mathrm{La}_{3-x} \mathrm{Ca}_{x} \mathrm{Te}_{4}$ as determined by the Hall effect compared to WDS and XRD. Ideal matching to the Hall carrier concentration is shown as the dashed line. 
coefficient for high carrier concentration compositions. The low mobility results in a low Hall coefficient and lower signalto-noise ratio limiting the accuracy of the results as observed as deviation from other analytical techniques.

At low carrier concentrations, it was observed that the samples tarnished more quickly, possibly due to the higher calcium content, which resulted in higher contact resistances to the samples. In addition, the higher resistivity of the low carrier concentration samples resulted in a greater challenge in obtaining Ohmic contact to the sample without metallization. These factors combine to result in the greater observed deviation between the Hall and WDS carrier concentrations for the low carrier concentration composition $n_{\mathrm{H}}=0.2 \times 10^{21} \mathrm{~cm}^{-3}$.

The deviation of the carrier concentration as determined by XRD from Hall carrier concentration at $n_{\mathrm{H}}=1.3 \times 10^{21} \mathrm{~cm}^{-3}$ can be attributed to the poorer refinement of the $x=0.775$ composition as indicated by the larger Bragg residual. The good agreement with Hall carrier concentration with the carrier concentration as calculated from the WDS composition suggests that the error originates from the Rietveld refinement of this composition.

The temperature dependence of the electrical resistivity is shown in Fig. 6. The measurements are labeled by their Hall carrier concentration $\left(\times 10^{21} \mathrm{~cm}^{-3}\right)$. The compositions of $\mathrm{La}_{3-x} \mathrm{Ca}_{x} \mathrm{Te}_{4}$ with carrier concentrations greater than $0.5 \times 10^{21} \mathrm{~cm}^{-3}$ exhibit typical heavily-doped semiconductor behavior. The resistivities of samples are low (less than $4 \mathrm{~m} \Omega \mathrm{cm}$ ) near the optimum carrier concentration and rise with temperature due to greater electronphonon scattering. The curve of the heavily Ca-doped low carrier concentration sample $\left(0.2 \times 10^{21} \mathrm{~cm}^{-3}\right)$ indicates thermally activated carriers with a decreasing resistivity up to $\sim 500 \mathrm{~K}$. A similar behavior is seen for lower carrier concentration samples of $\mathrm{La}_{3-x} \mathrm{Te}_{4}\left(0.1 \times 10^{21} \mathrm{~cm}^{-3}\right)$. However, there is no thermal activation of carriers for samples with $\geq 0.5 \times 10^{21} \mathrm{~cm}^{-3}$.

The calcium-doped samples follow similar trends to $\mathrm{La}_{3-x} \mathrm{Te}_{4}$ (dashed lines) for samples of comparable carrier concentrations previously reported by May et al. ${ }^{7}$ The resistivities of samples with carrier concentrations of $0.8 \times 10^{21} \mathrm{~cm}^{-3}$ and $2.1 \times 10^{21} \mathrm{~cm}^{-3}$

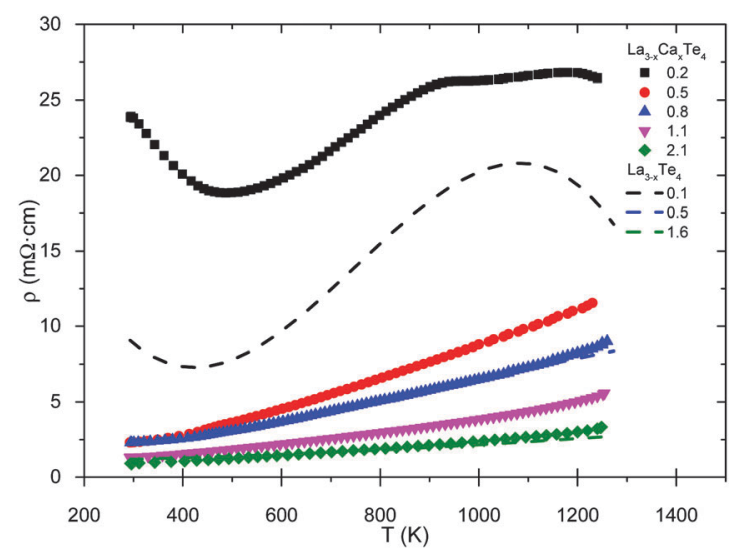

Fig. 6 High temperature resistivity of $\mathrm{La}_{3-x} \mathrm{Ca}_{x} \mathrm{Te}_{4}$ as compared to values reported for $\mathrm{La}_{3-x} \mathrm{Te}_{4}{ }^{7}$ The samples are labeled by their carrier concentration $\left(\times 10^{21} \mathrm{~cm}^{-3}\right)$.

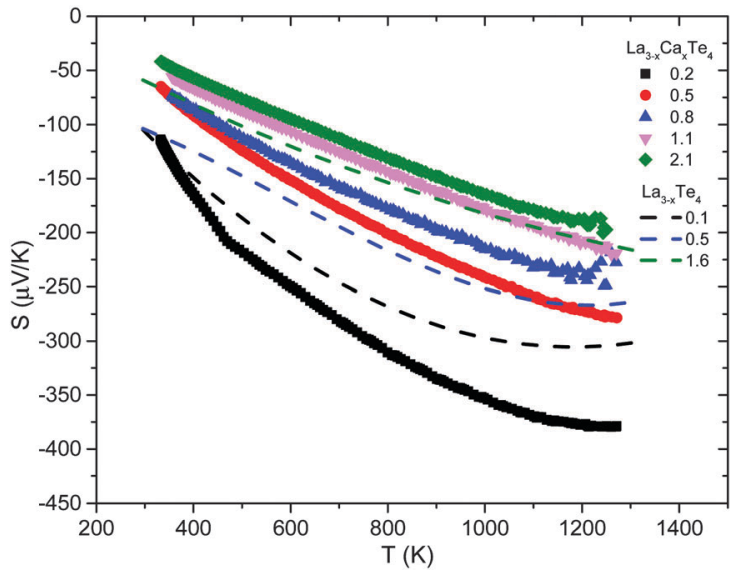

Fig. 7 High temperature Seebeck coefficient of $\mathrm{La}_{3-x} \mathrm{Ca}_{x} \mathrm{Te}_{4}$ as compared to values reported for $\mathrm{La}_{3-x} \mathrm{Te}_{4}{ }^{7}$ The samples are labeled by their carrier concentration $\left(\times 10^{21} \mathrm{~cm}^{-3}\right)$.

for $\mathrm{La}_{3-x} \mathrm{Ca}_{x} \mathrm{Te}_{4}$ overlay well with the $0.5 \times 10^{21} \mathrm{~cm}^{-3}$ and $1.6 \times$ $10^{21} \mathrm{~cm}^{-3}$ as reported by May et al. ${ }^{7}$ The differences in carrier concentration are likely due to the uncertainty in the measurement of carrier concentration as previously discussed.

The Seebeck coefficient as a function of temperature is presented in Fig. 7. The Seebeck coefficient decreases monotonically with increasing carrier concentration as expected. As previously explored by May et al., the presence of heavily doped bands near the conduction band edge enables the maintenance of a high Seebeck coefficient even at high dopant concentrations. ${ }^{9}$ This key property of $\mathrm{La}_{3-x} \mathrm{Te}_{4}$, which enables the material to be a high zT thermoelectric material, is still present for $\mathrm{La}_{3-x} \mathrm{Ca}_{x} \mathrm{Te}_{4}$.

The thermal bandgap for the material can be estimated from the maximum Seebeck coefficient and the temperature at which that occurs using the equation $E_{\mathrm{g}} \approx 2 e S_{\max } T_{\max } \cdot{ }^{18}$ The bandgap ranges from $\sim 0.45-0.95 \mathrm{eV}$ depending on the dopant concentration. These values agree with the bandgap of $\sim 0.8 \mathrm{eV}$ previously reported for $\mathrm{La}_{3-x} \mathrm{Te}_{4}{ }^{7}$

The Seebeck coefficient of $\mathrm{La}_{3-x} \mathrm{Ca}_{x} \mathrm{Te}_{4}$ as a function of temperature has the same temperature dependence as $\mathrm{La}_{3-x} \mathrm{Te}_{4}$. The desired effect was to modify the band structure of $\mathrm{La}_{3-x} \mathrm{Te}_{4}$ through calcium doping. To fully compare the effects of calcium doping, a plot of Seebeck coefficient versus electrical resistivity at $1275 \mathrm{~K}$ of $\mathrm{La}_{3-x} \mathrm{Ca}_{x} \mathrm{Te}_{4}$ compared to $\mathrm{La}_{3-x} \mathrm{Te}_{4}$ is provided in Fig. 8. The Seebeck coefficient with respect to the electrical resistivity exhibits essentially no difference between $\mathrm{La}_{3-x} \mathrm{Ca}_{x} \mathrm{Te}_{4}$ and $\mathrm{La}_{3-x} \mathrm{Te}_{4}$. A similar behavior was observed for the previously studied $\mathrm{La}_{3-x} \mathrm{Yb}_{x} \mathrm{Te}_{4} \cdot{ }^{17}$ These results suggest that the cation dopants are behaving as substitutional dopants which provide carriers to the structure, but are possibly at a level too low to affect the band structure of $\mathrm{La}_{3-x} \mathrm{Te}_{4}$ despite the $\sim 30$ at $\%$ substitution of the cation site by calcium. This result suggests that near the conduction band edge, the $5 \mathrm{~d}$ lanthanum atom orbitals are still controlling the transport phenomena.

Given that $\mathrm{La}_{3-x} \mathrm{Ca}_{x} \mathrm{Te}_{4}$ appears to exhibit a similar transport behavior the band structure near the conduction band is 


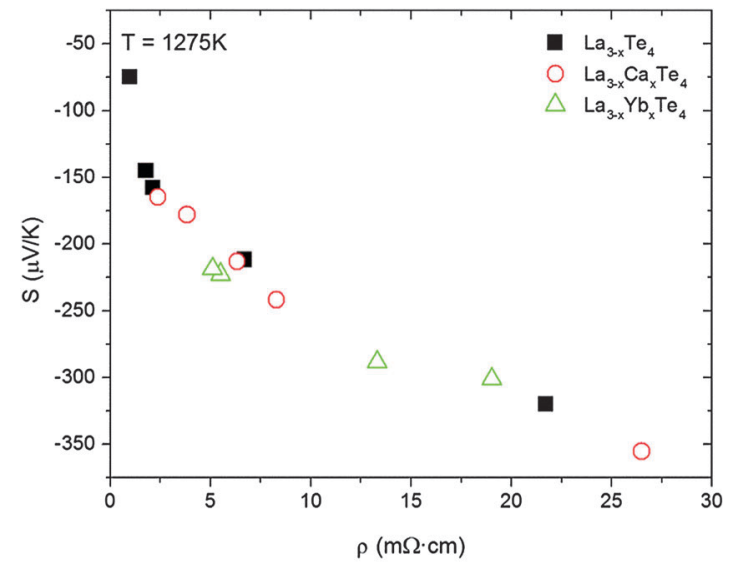

Fig. 8 The Seebeck coefficient of $\mathrm{La}_{3-x} \mathrm{Ca}_{x} \mathrm{Te}_{4}$ as a function of electrical resistivity follows a similar trend compared to $\mathrm{La}_{3-x} \mathrm{Te}_{4}$ and $\mathrm{La}_{3-x} \mathrm{Yb}_{x} \mathrm{Te}_{4}$ suggesting that calcium doping does not modify the band structure at $1275 K^{7,17}$

likely similar. May et al. previously found that $\mathrm{La}_{3-x} \mathrm{Te}_{4}$ fits a multiparabolic band model and derived the effective masses of $m_{1}{ }^{*} / m_{\mathrm{e}}=0.39, m_{2}{ }^{*} / m_{\mathrm{e}}=1.05$, and $m_{3}{ }^{*} / m_{\mathrm{e}}=1.56$ with corresponding band degenerancies of $g_{1}=2, g_{2}=1$, and $g_{3}=2 .{ }^{9}$

The resulting electronic transport properties are in agreement with the results from X-ray diffraction and electron microprobe WDS in that the calcium atoms are substituting into the structure as an unambiguous +2 cation. Through calcium substitution, a finer control over carrier concentration is realized. The $\mathrm{La}^{3+}: \mathrm{Ca}^{2+}$ composition can be adjusted to only add one electron at a time to the system, whereas the vacancy doped system adds three. This result supports the initial hypothesis for enhanced control over carrier concentration as variations in carrier concentration progress more gradually as a function of stoichiometry. Fine carrier concentration control is important for the $\mathrm{La}_{3-x} \mathrm{Te}_{4}$ system as small deviations near the optimized carrier concentration result in significant changes in the thermoelectric performance.

\section{Thermal transport properties}

The thermal conductivity as a function of temperature of $\mathrm{La}_{3-x} \mathrm{Ca}_{x} \mathrm{Te}_{4}$ compared to $\mathrm{La}_{3-x} \mathrm{Te}_{4}$ is shown in Fig. 9. Paralleling the electronic properties, the total thermal conductivity is similar between $\mathrm{La}_{3-x} \mathrm{Ca}_{x} \mathrm{Te}_{4}$ and the vacancy controlled $\mathrm{La}_{3-x} \mathrm{Te}_{4}$ at equivalent carrier concentrations. The total thermal conductivities for the various compositions are $6-14 \mathrm{~mW} \mathrm{~cm}^{-1} \mathrm{~K}^{-1}$ at $1275 \mathrm{~K}$ with the thermal conductivities increasing with increasing carrier concentration. This proportional relation suggests a significant contribution of the electron thermal conductivity $\kappa_{\mathrm{e}}$ for the high carrier concentration samples. The relative proportions are analyzed by parsing the contributions to total thermal conductivity.

The total thermal conductivity is the sum of the lattice and electronic thermal conductivity: $\kappa_{\mathrm{t}}=\kappa_{\mathrm{e}}+\kappa_{\mathrm{l}}$. To determine the lattice thermal conductivity, the electronic contribution to the total

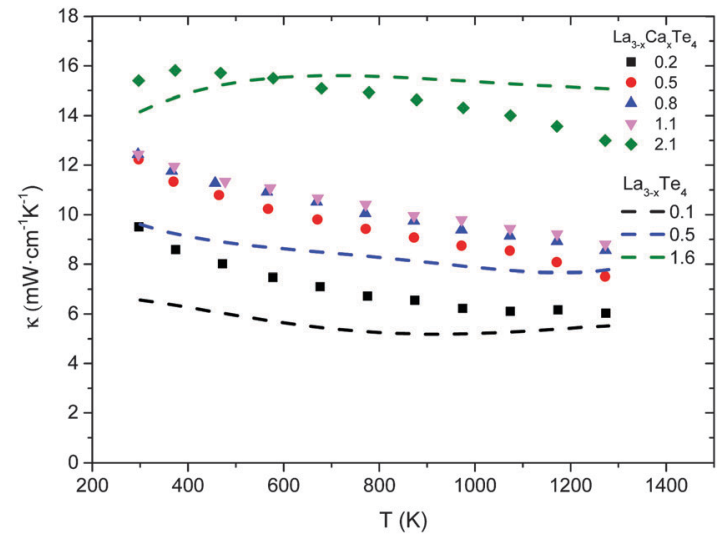

Fig. 9 Thermal conductivity as a function of temperature for $\mathrm{La}_{3-x} \mathrm{Ca}_{x} \mathrm{Te}_{4}$ as compared to $\mathrm{La}_{3-x} \mathrm{Te}_{4}{ }^{7}$

thermal conductivity was first calculated using the WiedemannFranz law $\kappa_{\mathrm{e}}=\sigma L T$ where $\sigma$ is the electrical conductivity, $L$ is the Lorenz number, and $T$ is the absolute temperature, followed by subtracting from $\kappa_{\mathrm{t}}$. The Lorenz number is calculated as a function of Seebeck coefficient using the approximation $L=1.5+\exp (-|S| / 116) .{ }^{19}$ The Seebeck coefficient varies as a function of temperature and carrier concentration resulting in Lorenz numbers in the range of $2.2-1.5 \times 10^{21} \mathrm{~W} \Omega \mathrm{K}^{-2}$.

The lattice thermal conductivity as a function of temperature is shown in Fig. 10. The minimum lattice thermal conductivity is achieved at a carrier concentration of $1.1 \times 10^{21} \mathrm{~cm}^{-3}$ and $0.2 \times$ $10^{21} \mathrm{~cm}^{-3}$ for the calcium-doped system. The lattice thermal conductivity is comparable to the lattice thermal conductivity for $\mathrm{La}_{3-x} \mathrm{Te}_{4}$ which ranges from $4-8 \mathrm{~mW} \mathrm{~cm}^{-1} \mathrm{~K}^{-1}$ at $1000 \mathrm{~K}^{7}$ The intrinsically low lattice thermal conductivities for all compositions can be attributed to the complex crystal structure resulting in significant Umklapp scattering of acoustic phonons. ${ }^{20}$ Further reduction of lattice thermal conductivity at higher carrier concentrations can be attributed to electron-phonon scattering which can be appreciable for material with carrier concentrations of $\sim 10^{21} \mathrm{~cm}^{-3} \cdot{ }^{21}$ Reduced lattice thermal conductivities at low carrier concentrations can be attributed to the higher

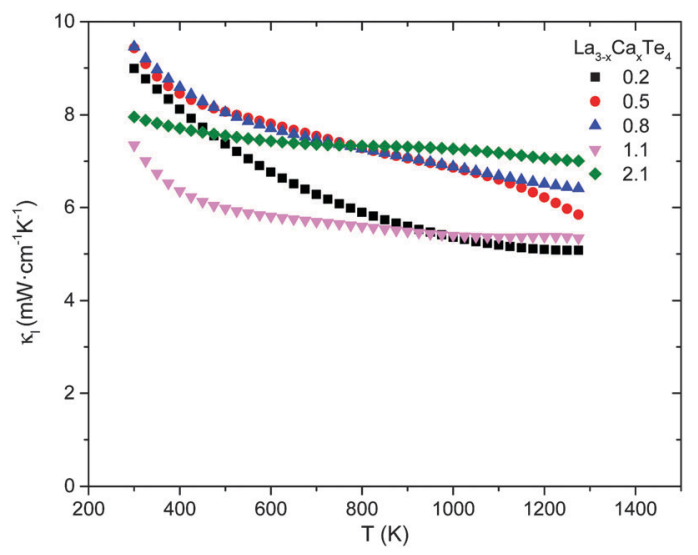

Fig. 10 Lattice thermal conductivity as a function of temperature for $\mathrm{La}_{3-x} \mathrm{Ca}_{x} \mathrm{Te}_{4}$. 
defect concentrations leading to enhanced point-defect scattering. The effects of point-defect scattering and electron-phonon scattering are small considering the lattice thermal conductivity varies between 6-7.5 $\mathrm{mW} \mathrm{cm}^{-1} \mathrm{~K}^{-1}$ despite the order of magnitude change in carrier concentration and 30 at\% substitution of the lanthanum positions with calcium suggesting that Umklapp scattering is still the dominant scattering mechanism. The effect of point-defect scattering can potentially be more significant if the substituting atom results in a greater lattice distortion. In the case of calcium doping, the lattice parameter change is less than $0.1 \%$.

The lattice thermal conductivity takes into account the bipolar contribution to thermal conductivity from thermally activated minority carriers which effect can be observed as a rise in the lattice thermal conductivity at high temperature. However, in the case of $\mathrm{La}_{3-x} \mathrm{Ca}_{x} \mathrm{Te}_{4}$ and $\mathrm{La}_{3-x} \mathrm{Te}_{4}$ near the optimized carrier concentration, the Fermi level is in the conduction band away from the conduction band edge and the material has a sufficient band gap, thereby limiting the effect of minority carriers. Therefore the lattice thermal conductivity is relatively flat as a function of temperature which contributes, in conjunction with greater electron-phonon scattering, to a flat total thermal conductivity at increased temperatures.

\section{Thermoelectric figure of merit}

The end result of calcium substitution in $\mathrm{La}_{3-x} \mathrm{Te}_{4}$ is that there is a marginal improvement in the thermoelectric figure of merit zT as shown in Fig. 11. The peak zT of 1.2 at $1275 \mathrm{~K}$ was obtained at the optimum doping level of $1.1 \times 10^{21} \mathrm{~cm}^{-3}$ which is $\sim 10 \%$ improvement compared to vacancy controlled $\mathrm{La}_{3-x} \mathrm{Te}_{4}$ $\mathrm{zT} \sim 1$ 1.1. This minimal improvement is expected as it appears that calcium is doping into the $\mathrm{La}_{3-x} \mathrm{Te}_{4}$ structure without much effect upon the band structure. The improvement to zT is thereby attributed to better optimization of carrier concentration enabled by substitution with a divalent cation. This result is reflected as well in the optimization of $\mathrm{zT}$ as a function of carrier concentration as shown in Fig. 12. The behavior of $\mathrm{La}_{3-x} \mathrm{Ca}_{x} \mathrm{Te}_{4}$

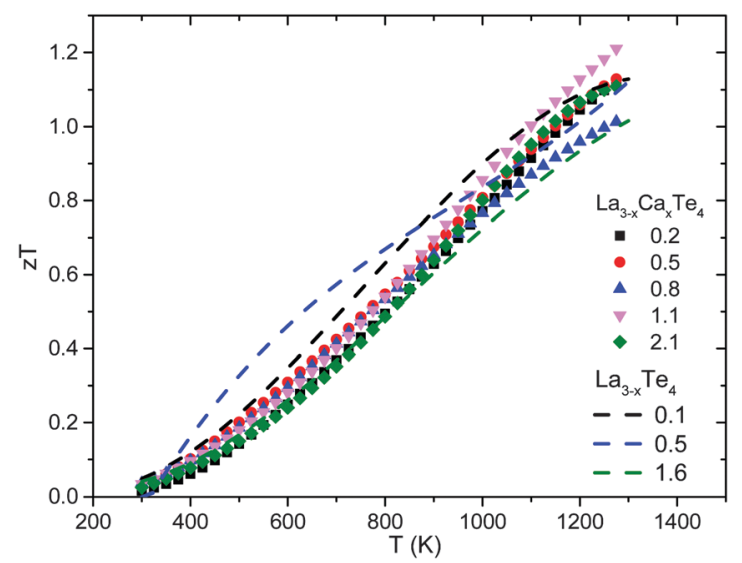

Fig. 11 The thermoelectric figure of merit $z T$ as a function of temperature for $\mathrm{La}_{3-x} \mathrm{Ca}_{x} \mathrm{Te}_{4}$ as compared to $\mathrm{La}_{3-x} \mathrm{Te}_{4}{ }^{7}$

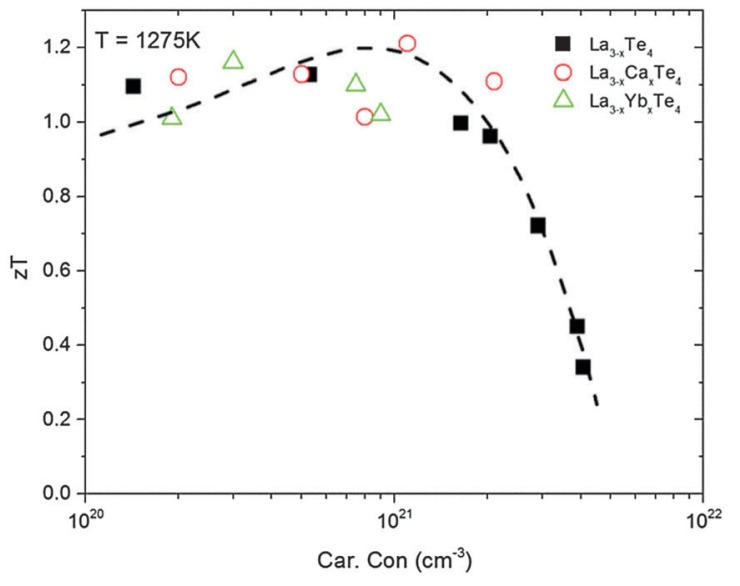

Fig. 12 The thermoelectric figure of merit zT at $1275 \mathrm{~K}$ as a function of carrier concentration for $\mathrm{La}_{3-x} \mathrm{Ca}_{x} \mathrm{Te}_{4}$ as compared to $\mathrm{La}_{3-x} \mathrm{Te}_{4}$ and $\mathrm{La}_{3-x} \mathrm{Yb}_{x} \mathrm{Te}_{4}{ }^{7,17}$ The results fit well to the multi-parabolic band model of $\mathrm{La}_{3-x} \mathrm{Te}_{4}$ (dashed line).

follows the multiparabolic band model developed for $\mathrm{La}_{3-x} \mathrm{Te}_{4}$. For comparison, results from the study on $\mathrm{La}_{3-x} \mathrm{Yb}_{x} \mathrm{Te}_{4}$ are overlayed and also fit the model well. ${ }^{17}$ Deviation from the curve is likely due to the difficulty in measuring the carrier concentration.

\section{Experimental}

\section{Materials and synthesis}

Samples were prepared using mechanochemical synthesis from the elements first described elsewhere. ${ }^{7}$ Metallic lanthanum (Hefa Rare Earth 99.95\%), calcium shot (Alfa Aesar 99.5\%), and tellurium lump (Alfa Aesar 99.999+\%) were sealed in a stainless steel ball mill vial with stainless steel balls (SPEX SamplePrep) in an argon glovebox. The materials were milled until they became a black fine homogeneous powder of the desired product. The powders were then compacted in graphite dies through spark plasma sintering (SPS) under vacuum at temperatures above $1200{ }^{\circ} \mathrm{C}$ and a pressure of $80 \mathrm{MPa}$ yielding samples with $>97 \%$ of theoretical density.

\section{X-ray diffraction}

X-ray diffraction data were collected on a Phillips PANalytical X'Pert Pro diffractometer with $\mathrm{Cu} \mathrm{K}$. radiation $\left(\lambda_{1}=1.540590 \AA\right.$, $\lambda_{2}=1.544310 \AA$ ), equipped with an X'Celerator detector. Data were collected over a $2 \theta$ range of $20-100^{\circ}$, with a $0.008^{\circ}$ step size and a time of $415 \mathrm{~s}$ per step. Sintered samples were ground and were mixed with a powdered silicon (Alfa Aesar, 325 mesh, 99.999\%) to provide an internal standard for an accurate lattice parameter determination. Due to the pyrophoric nature of powdered $\mathrm{La}_{3} \mathrm{Te}_{4}$, all measurements were performed using a $7.5 \mu \mathrm{m}$ thick Kapton film to seal the sample on a silicon zero background holder. Rietveld refinements were performed with the FullProf program. ${ }^{22}$ The cubic space group $I \overline{4} 3 d$ and the structural data of Cox et al. ${ }^{14}$ were used as the initial structural model, and the input for the various solid solutions were altered accordingly. An experimental uncertainty for the obtained lattice 
parameter of $0.001 \AA$ has been determined though the refinement of a $\mathrm{LaB}_{6}$ standard. A slightly larger uncertainty in the refined lattice parameter and occupancies can be expected in the sample with composition $\mathrm{La}_{2.225} \mathrm{Ca}_{0.775} \mathrm{Te}_{4}$, due to a smaller amount of sample in the beam. For a stable refinement of atomic occupancies, the Te occupancy was fixed to $100 \%$ as no Te vacancies are to be expected.

\section{Wavelength dispersive spectroscopy}

A JEOL Super Probe electron microprobe analyzer and wave dispersive spectroscopy (WDS) was used to examine elemental compositions. The standards used were Te metal, $\mathrm{LaPO}_{4}$, and $\mathrm{CaAl}_{2} \mathrm{Si}_{2} \mathrm{O}_{8}$ (Anorthite), for the respective elements. For each sample, $N \geq 10$ spots were analyzed and the results were averaged to determine the composition of the sample.

\section{Measurement of transport properties}

The thermoelectric transport properties of the samples were measured using both custom setups and commercial instrumentation described elsewhere. ${ }^{23,24}$ A Netzsch LFA 457 was used to conduct laser flash thermal diffusivity measurements for determination of the thermal conductivity. The thermal conductivity is calculated through the relation $\kappa=D_{\mathrm{T}} C_{\mathrm{p}} d$ where $D_{\mathrm{T}}$ is the thermal diffusivity, $C_{\mathrm{p}}$ is the specific heat, and $d$ is the density. $D_{\mathrm{T}}$ is measured through laser flash thermal diffusivity and heat capacity is taken from previously published differential scanning calorimetry results which has been modified to add calcium content following the Dulong-Petit law. ${ }^{10}$ The density of the sample is corrected for the thermal expansion of the material as a function of temperature.

\section{Conclusions}

The successful doping of $\mathrm{La}_{3-x} \mathrm{Te}_{4}$ with $\mathrm{Ca}^{2+}$ was achieved through mechanochemical synthesis and indicates that substitutions of $\mathrm{Ca}^{2+}$ for $\mathrm{La}^{3+}$ is possible for a wide range of carrier concentrations. The level of $\mathrm{Ca}^{2+}$ doping was controlled by stoichiometry and confirmed by a combination of powder X-ray diffraction, wave dispersive spectroscopy, and electronic measurements. General behavior of the material is similar to that of vacancy doped $\mathrm{La}_{3-x} \mathrm{Te}_{4}$ first described by May et al. ${ }^{13}$ A more comprehensive set of calcium-substituted samples, with and without lanthanum vacancies, offers a future opportunity to ascertain the role of vacancies versus dopants as a phonon scattering site.

Improvement to zT was achieved through finer control of carrier concentration made possible by divalent cation substitution. The calcium atoms have little effect upon the band structure and drastic improvements to zT were not observed.

However, the high doping levels with calcium and its chemical difference to lanthanum suggest that $\mathrm{La}_{3-x} \mathrm{Te}_{4}$ is conducive to substitution with a wide range of cationic dopants. Furthermore, substitution of lanthanum with calcium or other cation dopants could be a promising avenue to reduce the cost of this material for commercial applications in light of increasing rare earth prices. The chemical flexibility of the structure suggests many possibilities for the improvement upon the already high zT of this high temperature thermoelectric material.

\section{Acknowledgements}

The authors would also like to thank Frank T. Kyte (Earth and Space Science Institute, UCLA) for his assistance in electron microprobe analysis. This work was performed at the Jet Propulsion Laboratory, California Institute of Technology under contract with the National Aeronautics and Space Administration. This work was supported by the NASA Science Missions Directorate's Radioisotope Power Systems Technology Advancement Program. Support was also provided in part by NSF IGERT: Materials Creation Training Program (MCTP) - DGE-0654431, the California NanoSystems Institute, EFRC Solid-State Solar-Thermal Energy Conversion Center (S3TEC) award number DE-SC0001299, the Summer Undergraduate Research Fellowship (SURF) through CalTech and JPL, and the UCLA MSD Scholars Program.

\section{Notes and references}

1 G. L. Bennett, in CRC Handbook of Thermoelectrics, ed. D. M. Rowe, 1995, pp. 515-537.

2 G. Schmidt, L. Dudzinski and T. Sutliff, in Radioisotopes Applications in Physical Sciences, ed. N. Singh, InTech, Reston, Virigina, 2011, pp. 419-456.

3 J.-P. Fleurial, JOM, 2009, 61, 79-85.

4 J. Yang and T. Caillat, MRS Bull., 2006, 31, 224-229.

5 G. J. Snyder and E. S. Toberer, Nat. Mater., 2008, 7, 105-114.

6 S. Bux, J.-P. Fleurial, T. Caillat, B. Y. Li, K. Star, S. Firdosy, V. Ravi, C.-K. Huang, B. Cheng, P. Gogna, J. M. Ma, P. von Allmen and T. Vo, 11th Int. Energy Convers. Eng. Conf., 2013.

7 A. May, J. Fleurial and G. Snyder, Phys. Rev. B: Condens. Matter Mater. Phys., 2008, 78, 1-12.

8 A. May, J. Snyder and J. Fleurial, Sp. Technol. Appl. Int. Forum, 2008, 672-678.

9 A. May, D. Singh and G. Snyder, Phys. Rev. B: Condens. Matter Mater. Phys., 2009, 79, 1-4.

10 O. Delaire, A. F. May, M. A. Mcguire, W. D. Porter, M. S. Lucas, M. B. Stone, D. L. Abernathy, V. A. Ravi, S. A. Firdosy and G. J. Snyder, Phys. Rev. B: Condens. Matter Mater. Phys., 2009, 80, 1-9.

11 T. Vo, P. von Allmen, C.-K. Huang, J. Ma, S. Bux, J.-P. Fleurial, P. Von Allmen, C.-K. Huang, J. Ma, S. Bux, J.-P. Fleurial, T. Vo, P. Von Allmen, C.-K. Huang, J. Ma and S. Bux, J. Appl. Phys., 2014, 116, 133701-133706.

12 A. F. May, J.-P. Fleurial and G. J. Snyder, Chem. Mater., 2010, 22, 2995-2999.

13 A. May, J.-P. Fleurial and G. Snyder, Phys. Rev. B: Condens. Matter Mater. Phys., 2008, 78, 1-12.

14 W. L. L. Cox, H. Steinfink and W. F. F. Bradley, Inorg. Chem., 1966, 5, 318-319.

15 F. L. Carter, J. Solid State Chem., 1972, 5, 300-313.

16 R. D. Shannon, Acta Crystallogr., Sect. A: Cryst. Phys., Diffr., Theor. Gen. Crystallogr., 1976, 32, 751-767. 
17 A. F. May, J.-P. Fleurial and G. J. Snyder, Chem. Mater., 2010, 22, 2995-2999.

18 H. J. Goldsmid and J. W. Sharp, J. Electron. Mater., 1999, 28, 869-872.

19 H.-S. Kim, Z. M. Gibbs, Y. Tang, H. Wang and G. J. Snyder, APL Mater., 2015, 3, 041506.

20 R. Viennois, K. Niedziolka and P. Jund, Phys. Rev. B: Condens. Matter Mater. Phys., 2013, 88, 174302.
21 X. Shi, Y. Pei, G. J. Snyder and L. Chen, Energy Environ. Sci., 2011, 4, 4086.

22 J. Rodrigues-Carvajal, Phys. B, 1993, 192, 55-69.

23 K. A. Borup, E. S. Toberer, L. D. Zoltan, G. Nakatsukasa, M. Errico, J. P. Fleurial, B. B. Iversen and G. J. Snyder, Rev. Sci. Instrum., 2012, 83, 123902.

24 C. Wood, D. Zoltan and G. Stapfer, Rev. Sci. Instrum., 1985, 56, 719-722. 